\title{
CARACTERIZAÇÃO MAGNÉTICA DOS EFEITOS DE DEFORMAÇÃO EM AÇOS ESTRUTURAIS ATRAVÉS DA TÉCNICA BITTER MODIFICADA*
}

\section{Resumo}

Lucas Kling e Silva ${ }^{1}$

Paula Mendes Jardim² Gabriela Ribeiro Pereira ${ }^{3}$ Nadja Sontag 4 Birgit Skrotzki ${ }^{5}$

A deformação plástica de aços acompanha mudanças irreversíveis da microestrutura, que podem ser consideradas como o estágio inicial do processo de fratura. No entanto, detectar, monitorar e avaliar previamente, estados de dano e pequenos defeitos, de forma não destrutiva, ainda se mostra desafiador. Foi reportado na literatura o fenômeno do surgimento espontâneo de fracos campos magnéticos em aços estruturais e em oleodutos, que são originados devido a tensões mecânicas heterogêneas ou térmicas. Esta observação não é associada com transformações de fase induzidas por deformação e parece ser uma ferramenta promissora para a caracterização prévia de danos em aços ferromagnéticos. Para proporcionar uma melhor compreensão das bases físicas deste processo, foram observadas as microestruturas magnéticas de tais materiais e as mudanças ocorridas nos domínios magnéticos dos mesmos após sofrerem deformação plástica. Para isto foi utilizado um meio de solução coloidal com partículas superparamagnéticas na escala nanométrica (ferrofluido) que proporcionou, através da técnica Bitter, observar não apenas uma mudança de tamanho dos domínios magnéticos do material, mas também modificações em sua morfologia. Foram estudados neste trabalho aços ferríticos com as respectivas concentrações de carbono em sua composição: $0.08 \%$; $0.22 \%$ e $0.45 \%$.

Palavras-chave: Domínios magnéticos; Aços; Ferrofluido; Deformação plástica.

\section{MAGNETIC CHARACTERIZATION FROM THE EFFECTS OF DEFORMATION ON STRUCTURAL STEELS USING THE MODIFIED TECHNIQUE BITTER}

\section{Abstract}

The plastic deformation results in irreversible microstructure changes in the steel, which can be considered as the initial stage of the fracture process. However, detecting, monitoring and evaluating, damage states and small defects nondestructively in advance still proves challenging. It was reported in literature the phenomenon of the spontaneous emergence of weak magnetic fields in structural steels and pipelines, which originates due to heterogeneous mechanical and / or thermal stresses. This observation is not associated with induced phase transformations by deformation and appears to be a promising tool for the prior characterization of damage in ferromagnetic steels. To provide a better understanding of the physical bases of the process, the magnetic microstructure of such materials and the change of magnetic domains after undergoing plastic deformation were studied. For this purpose, a colloidal solution with paramagnetic particles in the nanometer scale (ferrofluid) was used, through the Bitter technique, in order to, not only observe a change in size of the magnetic domains of the material, but also changes in their morphology. Ferritic steels with different carbon contents $(0.08 \% ; 0.22 \%$ and $0.45 \%)$ were studied in this work.

Keywords: Magnetic domains; Steels; Ferrofluid; Plastic deformation. 
1 Engenheiro Metalúrgico, Mestrando em engenharia metalúrgica, Departamento de Engenharia Metalúrgica e Materiais, Universidade federal do Rio de Janeiro - UFRJ, Rio de Janeiro, Rio de Janeiro, Brasil.

2 Doutora em Engenharia de materiais e de processos químicos, Professora Doutora Engenheira, Pesquisadora, Universidade federal do Rio de Janeiro - UFRJ, Rio de Janeiro, Rio de Janeiro, Brasil.

3 Doutora em Ciências e Engenharia Nuclear, Professora Doutora Física, Pesquisadora, Universidade federal do Rio de Janeiro - UFRJ, Rio de Janeiro, Rio de Janeiro, Brasil.

4 Doutoranda em Engenharia de Materiais, Mestra, pesquisadora, Fachbereich 5.2 Experimentelle und modellbasierte Werkstoffmechanik, Bundesanstalt für Materialforschung und -prüfung, Berlin, Alemanha.

5 Doutora em Engenharia de Materiais, Professora Doutoura Engenheira, Chefe de departamento, Fachbereich 5.2 Experimentelle und modellbasierte Werkstoffmechanik, Bundesanstalt für Materialforschung und -prüfung, Berlin, Alemanha. 


\section{INTRODUÇÃO}

Os principais requisitos para os aços destinados à aplicação estrutural são: elevada tensão de escoamento, elevada tenacidade, boa soldabilidade, homogeneidade microestrutural, susceptibilidade de corte por chama sem endurecimento e boa trabalhabilidade em operações tais como corte, furação e dobramento, sem que se originem fissuras ou outros defeitos [1].

Os aços estruturais que são usados sob tensão mecânica podem ter sua microestrutura modificada devido às deformações plásticas ou elásticas. As propriedades magnéticas desses aços são bastante sensíveis a mudanças em suas microestruturas. Essas mudanças magnéticas podem ser detectadas por técnicas não-destrutivas, avaliando a mudança dos domínios magnéticos. Desta forma, a avaliação da qualidade e a detecção prévia de defeitos nestes materiais durante operação torna-se de crucial importância [2].

Dubov [3] relatou o surgimento espontâneo de fracos campos magnéticos em aços estruturais após estes serem submetidos a deformações plásticas. Tais campos emergem não devido a transformações de fases induzidas mecanicamente ou termicamente, mas sim por interações magneto-mecânicas. O surgimento destes campos magnéticos e a influência do efeito de topografia proveniente da deformação plástica foram estudados de forma a revelar que sua intensidade provém além de mudanças topográficas [4].

Kida [5] observou as mudanças nos campos magnéticos residuais durante o crescimento da fissura em torno da área de deformação plástica e relatou que os campos magnéticos se movem à medida que a área plástica se expande, os campos magnéticos mudam na frente das pontas de fissura e existe uma forte correlação entre a diminuição das intensidades do campo magnético e as faixas do fator de intensidade de tensão.

Sonntag e Pelker [6, 7], a partir do princípio de magneto resistência gigante, criaram sensores onde podem medir pequenas flutuações na intensidade de campo magnético nos materiais. Desta forma percebeu-se que após a deformação plástica de aços de construção há uma acentuada modificação nas informações magnéticas deste material.

Talonen [8] utilizou o ferrofluido, um líquido coloidal com partículas superparamagnéticas em escala nanométrica, para observação e quantificação de martensita ferromagnética em aços austeníticos metaestáveis que foram submetidos à deformação plástica.

No presente trabalho, o objetivo é investigar a influência da deformação plástica na distribuição e magnitude do campo magnético dos aços ASTM A366, ASTM A283C e ASTM 1045, respectivamente com $0.08 \%, 0.22 \%$ e $0.48 \%$ de carbono, com 0 intuito de compreender os mecanismos magneto-físicos responsáveis por estas mudanças, utilizando a técnica Bitter através do ferro fluido. O ferrofluido e correlatos já foram utilizados por Talonen [8], Quintana [9] e Bitter [10] para a obtenção de resposta magnética de materiais, sem a influência de campos magnéticos externos.

Dado o conjunto de fatos mencionados, este trabalho primeiramente sistematizou e metodizou a utilização e aplicação do ferrofluido para os aços investigados, seguido de um estudo para a compreensão da informação magnética revelada pelo ferrofluido e sua correlação com a microestrutura dos materiais. Posteriormente foi realizada a identificação e interpretação dos domínios magnéticos e, finalmente, a observação das modificações nestes domínios ocasionadas devido 
à deformação plástica. Para isso, foram investigadas as condições específicas para a escolha dos diferentes tipos de ferrofluido e foi determinado o grau de dissolução correta para os ferrofluidos e respectivos solventes que levaram aos resultados almejados. Dessa forma foi possível compreender e interpretar os domínios magnéticos dos aços estudados isentos de deformação plástica e as modificações sofridas pelos domínios magnéticos devido à deformação plástica.

\section{MATERIAIS E MÉTODOS}

Para a observação da microestrutura magnética dos materiais em questão foram experimentados previamente os ferrofluidos tanto das séries a base de água destilada, quanto os a base de óleo mineral, de forma que a diluição ideal encontrada foi de uma gota de ferrofluido para 160 gotas de água destilada e uma gota de ferrofluido para 60 gotas de óleo mineral [11].

A fim de observar as alterações nos domínios magnéticos após a deformação plástica, o seguinte experimento foi proposto: a primeira etapa foi a observação dos domínios magnéticos em áreas específicas dos materiais através do ferrofluido; a segunda etapa foi a realização de três endentações (nas áreas supracitadas) com uma esfera de $2,5 \mathrm{~mm}$ de diâmetro do tipo Brinnel, na diagonal das amostras, com idênticas cargas $(612,9 \mathrm{~N}$ com uma distância de 7,0mm entre si para evitar a zona de influência da deformação) para serem utilizadas como parâmetros de medida principal. Também foram realizadas duas endentações do tipo Vickers nas bordas das amostras para serem utilizadas como controle, de forma, a saber, a perda de material durante os processos metalográficos subsequentes; a terceira etapa consistiu na preparação metalográfica das amostras de modo a retirar as endentações por meio das lixas e polimentos até as amostras ficarem novamente planas. Após isto foi aplicado novamente o ferrofluido onde estavam as endentações (área anteriormente observada) e notou-se a alteração nos domínios magnéticos.

Para a realização deste estudo foram utilizados como amostras os aços que podem ser observados, junto com sua composição química, na Tabela 1.

Tabela 1: Composição dos aços estudados.

\begin{tabular}{|c|c|c|c|c|c|c|c|c|}
\hline ASTM & $\mathbf{C} \%$ & Mn\% & $\mathbf{P} \%$ & $\mathbf{S} \%$ & $\mathbf{C u} \%$ & Si & $\mathbf{C r} \%$ & Ni \\
\hline A366 & 0,08 & 0,60 & 0,03 & 0,04 & 0,2 & - & - & - \\
\hline A283C & 0,22 & 1,60 & 0,04 & 0,05 & - & 0,05 & - & - \\
\hline 1045 & 0,45 & 0,75 & 0,03 & 0,03 & - & 0,25 & - & - \\
\hline
\end{tabular}

As amostras dos aços descritos na Tabela 1 foram recebidas em chapas laminadas e foram cortadas utilizando-se um disco de corte 30A13 "Struers". Em seguida cada corpo de prova (CP) foi embutido utilizando-se pó de metil-metacrilato (“Dermotec 35"). Para a observação da informação magnética microestrutural, foram utilizados os ferrofluidos das séries EMG 308 e EMG 911 do fabricante "Ferrotec". Após o embutimento, os CP's foram submetidos aos processos metalográficos padrões. Para a caracterização microestrutural, os CP's já repreparados metalograficamente, foram atacados quimicamente, como pode ser observado na Tabela 2. 
Tabela 2: Composição química, temperatura, método e finalidade do ataque

\begin{tabular}{|l|l|l|l|l|}
\hline Ataque & $\begin{array}{l}\text { Composição } \\
\text { química }\end{array}$ & Método & Temperatura & Finalidade \\
\hline Nital & $\begin{array}{l}2 \mathrm{ml} \mathrm{HnO}_{3}+98 \mathrm{ml} \\
\mathrm{C}_{2} \mathrm{H}_{6} \mathrm{O}\end{array}$ & Imersão & $25^{\circ} \mathrm{C}$ & $\begin{array}{l}\text { Aços Carbono em } \\
\text { Geral }\end{array}$ \\
\hline Picral & $\begin{array}{l}5 \mathrm{gr} \text { Ácido Pícrico } \\
+100 \mathrm{ml} \mathrm{Álcool} \\
\text { Etílico }\end{array}$ & Imersão & $25^{\circ} \mathrm{C}$ & $\begin{array}{l}\text { Aços Carbono em } \\
\text { Geral tratados } \\
\text { termicamente }\end{array}$ \\
\hline
\end{tabular}

As micrografias deste trabalho foram realizadas no microscópio óptico Axioplan 2, da empresa ZEISS, que conta com uma câmera modelo UI2240SE-C de 1.0 megapixel e o software Pixelferber que foi utilizado para realizar quantificação nas imagens capturadas.

\section{RESULTADOS E DISCUSSÃO}

Serão apresentados a seguir os resultados referentes à observação e avaliação da informação magnética das amostras sem deformação plástica através de ensaios com ferrofluido, resultados representativos da correlação das microestruturas convencionais (fases e tamanhos de grão) com as informações magnéticas observadas através do ensaio com ferrofluido e, por fim, serão apresentados resultados, também representativos, das alterações da informação magnética macro e microestruturais das amostras após deformação plástica.

\subsection{Correlação da informação magnética com microestrutura convencional}

A seguir na Figura 1 (a) e (c) pode-se observar a imagem da informação magnética de uma amostra de aço ASTM A283C, onde as partículas superparamagnéticas possuem maior aglomeração em locais específicos além dos domínios magnéticos. Na figura 1 (b) e (d) temos a microestrutura convencional da mesma região das imagens (a) e (c). Destaca-se, em (a) e (b) uma região com grande aglomeração das partículas superparamagnéticas constituintes do ferrofluido. Ao analisar a figura 1 (b) e (d), observa-se que a região com grande aglomeração de partículas é, na verdade, a região onde se encontra perlita em forma de lamelas. Isto se deve ao fato do alto caráter ferromagnético da cementita, que é um constituinte da perlita. Este forte caráter ferromagnético da cementita é explicado por [12]. 


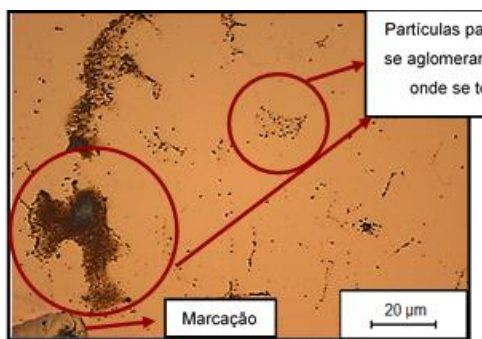

(a)

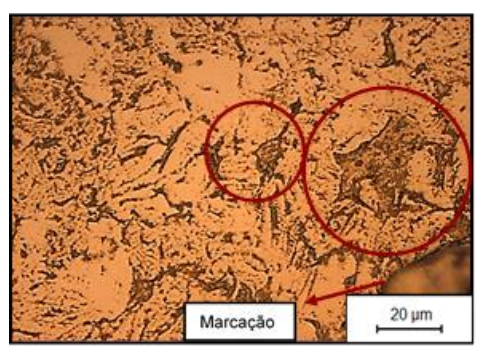

(c)

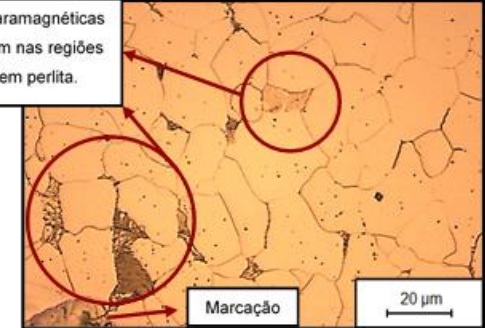

(b)

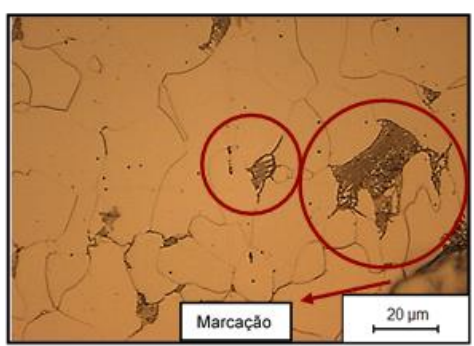

(d)

Figura 1: Imagens (a) e (c) representam a informação magnética (perlita e domínios magnéticos) da amostra ASTM A283C. (b) e (d) microestrutura convencional (perlita) da amostra ASTM A283C.

Portanto, percebe-se que o ensaio com ferrofluido é capaz de revelar não apenas as informações magnéticas provenientes dos domínios magnéticos da ferrita, como também as informações magnéticas provenientes dos microconstituintes do material.

Na figura 2 (a) pode-se observar a informação magnética de uma amostra de aço ASTM A366, onde é possível distinguir diferentes regiões em contraste. $\mathrm{Na}$ figura 2 (b) temos a imagem da mesma região, mas atacada com nital. Na figura 2 (d) foi destacado em azul os contornos de grão revelados na figura 2 (b). A figura 2 (c) é a sobreposição das linhas azuis da figura 2 (d) com a figura 2 (a), pois desta forma podemos correlacionar as regiões de contornos de grão com a informação magnética do aço em questão.

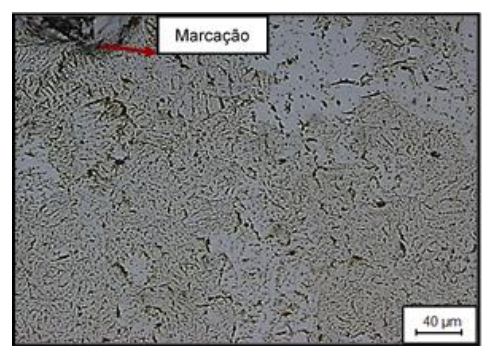

(a)

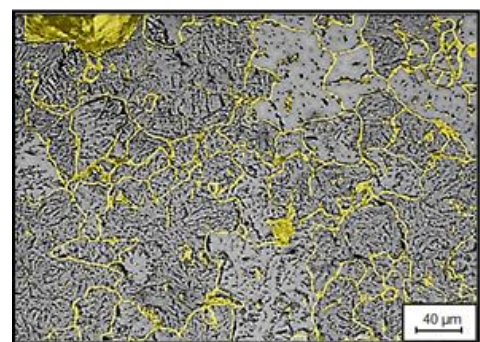

(c)

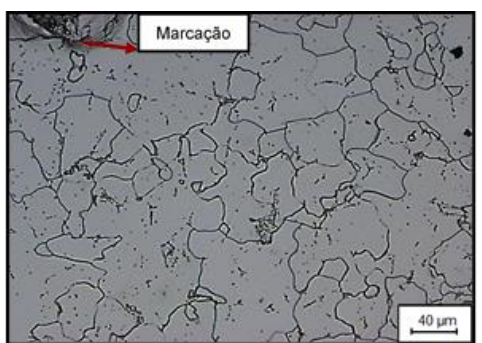

(b)

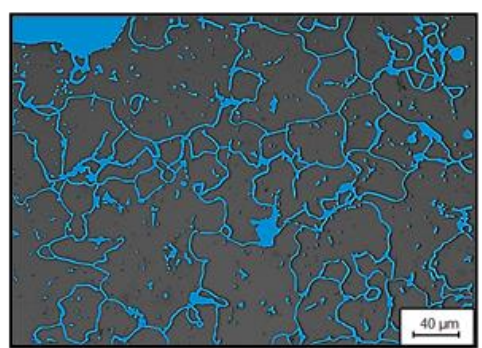

(d)

Figura 2: Em (a) tem-se a imagem da informação magnética de uma determinada região do aço ASTM A366. Em (b) tem-se a mesma região com a microestrutura revelada. Em (c) tem-se a sobreposição dos contornos na imagem da informação magnética. Em (d) tem-se o destaque dos contornos de grão. 
A sobreposição das linhas destes contornos de grão sobre a imagem (a) nos permite observar uma correlação do contraste da microestrutura magnética com os contornos de grão. Isto ocorre, pois, os contornos de grão são também áreas onde temos informação magnética, já que os contornos de grão também são interfaces entre dois ou mais domínios magnéticos, que se distribuem homogeneamente dentro dos grãos.

\subsection{Relação deformação domínios magnéticos}

\subsubsection{Caracterização dos aços, não deformados, com ferrofluido}

As partículas superparamagnéticas concentram-se sobre as paredes de Bloch, desta forma a distância entre duas listras ou linhas nas imagens acima significam um domínio magnético [13].

$\mathrm{Na}$ figura 3 (a) e (b) é possível observar claramente que os domínios magnéticos se apresentam de forma alongada e paralela. Na figura (c) e (d) observamos que os domínios magnéticos se apresentam mais curtos e as partículas paramagnéticas concentram-se não apenas nas paredes de Bloch, mas também em regiões específicas, como a perlita. Na figura (e) e (f) ainda podemos observar linhas paralelas que são as fronteiras dos domínios, porém com um pouco mais de dificuldade. Isto se deve ao fato de que esta amostra possui um maior teor de carbono em sua concentração, o que dificulta um pouco mais a observação dos domínios magnéticos devido à maior formação e concentração de fases com informação magnética, como por exemplo a perlita.

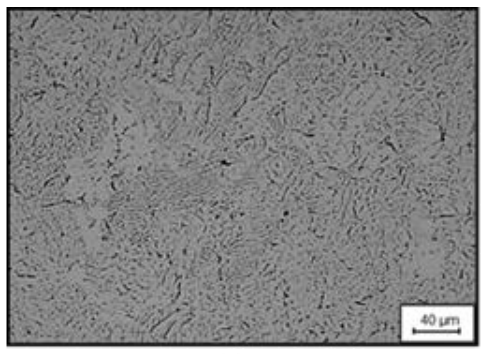

(a)

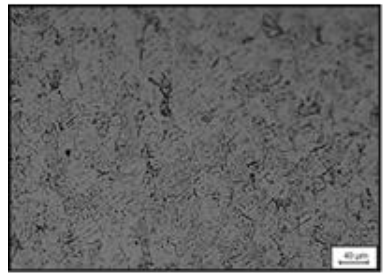

(c)

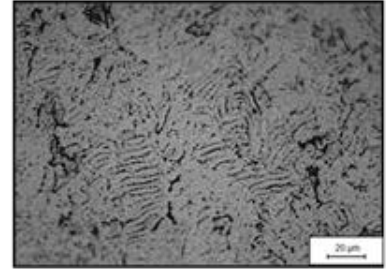

(d)

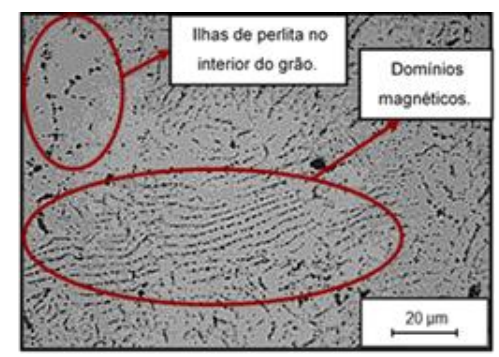

(b)
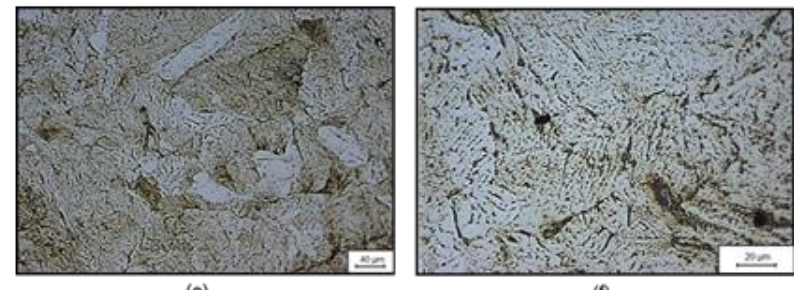

Figura 3: Domínios magnéticos do aço ASTM A366 com aumento de 100X em (a) e 200X em (b), do aço ASTM A283C com aumento de 100X em (c) e 200X em (d) e do aço ASTM 1045 com aumento de $100 \mathrm{X}$ em (e) e $200 \mathrm{X}$ em (f).

\subsubsection{Caracterização dos aços, deformados plasticamente, com ferrofluido}

A figura 4 mostra uma área macroscópica da amostra do aço ASTM A283C, que com o auxílio do ferrofluido se observa a área onde as endentações haviam sido 
feitas. As partículas superparamagnéticas aglomeram-se exatamente na área que foi deformada plasticamente. Isto reafirma que após deformação plástica ocorre uma alteração na intensidade de campos magnéticos da amostra.

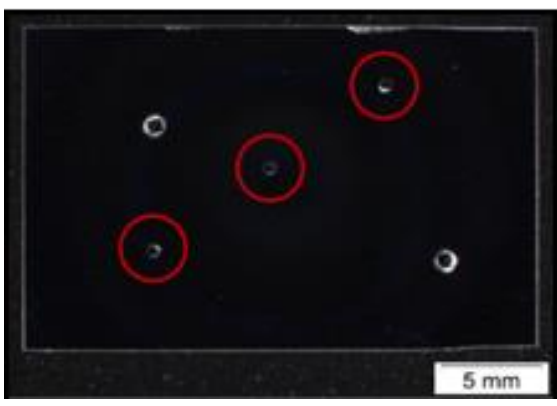

a)

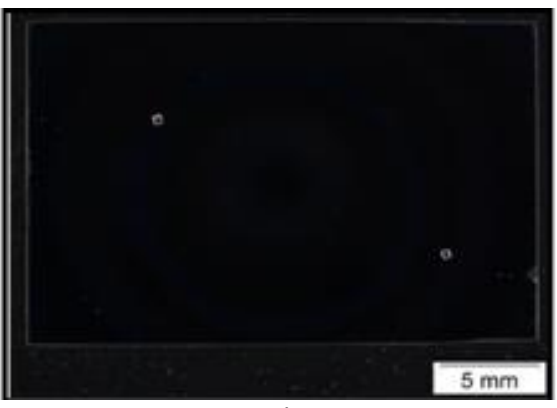

b)

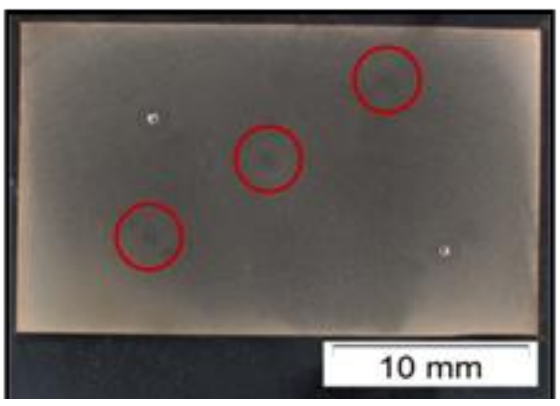

c)

Figura 4: Em (a) observa-se a amostra com as endentações, em (b) observa-se a amostra após as endentações do tipo Brinnel terem sido totalmente removidas e em (c) observa-se a informação magnética de (b), com auxílio do ferrofluido.

O mesmo experimento foi realizado para uma amostra do aço ASTM A366, porém foram realizadas endentações apenas do tipo Brinnel localizadas ao longo do comprimento da amostra. O resultado após ensaio com ferrofluido pode ser observado na Figura 5.

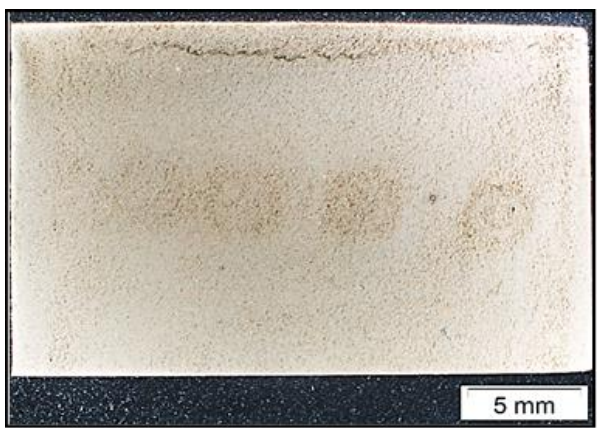

(a)

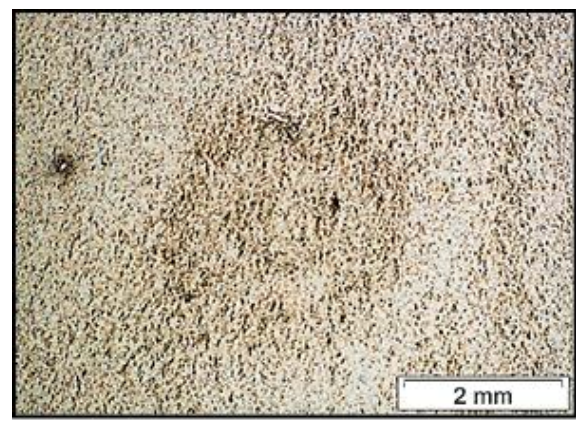

(b)

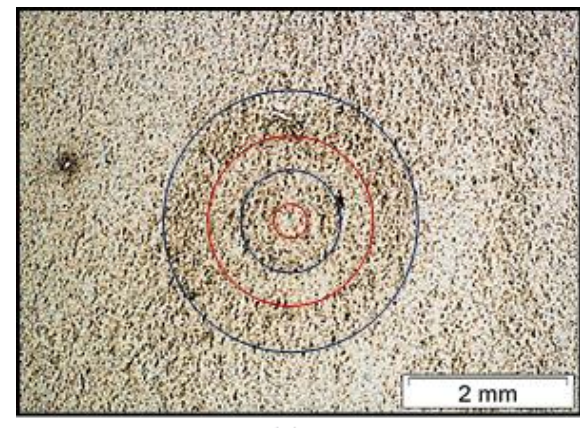

(c)

Figura 5: Em (a) tem-se resultado macroscópico da amostra ASTM A366 ensaiado com ferrofluido após endentações terem sido removidas. Em (b) tem-se região de uma das endentações em maior aumento. Em (c) tem-se a imagem (b) com delimitações destacando regiões claras e escuras.

$\mathrm{Na}$ figura 5 (a) tem-se, após ensaio com ferrofluido, claramente as regiões onde havia as endentações previamente. Ao observar estas regiões em maior aumento na imagem (b) observa-se a presença de áreas mais escuras, devido a uma maior aglomeração de ferrofluido, e áreas mais claras. Estas áreas foram destacadas na imagem (c) para a melhor observação do leitor.

$\mathrm{Na}$ figura 6 (a), (c), (e) e (g) tem-se os resultados de ensaio com ferrofluido nas regiões da figura 5 (a), antes de realizar as endentações. Isto foi feito para observar a informação magnética antes da deformação plástica. Já na figura 6 (b), (d), (f) e (h) tem-se imagens destas regiões após a deformação plástica, como visto na figura 5 (c), porém agora em maior aumento. 


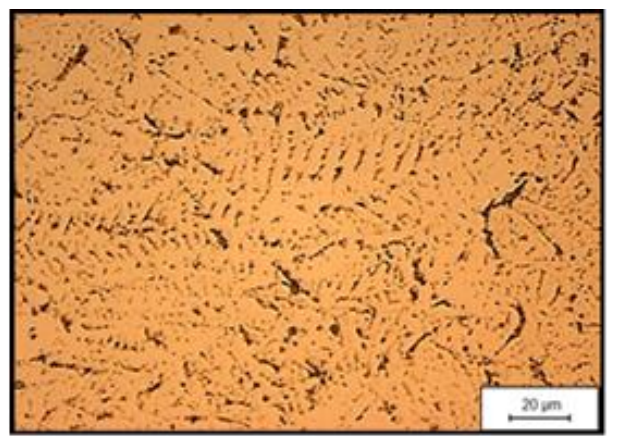

(a)

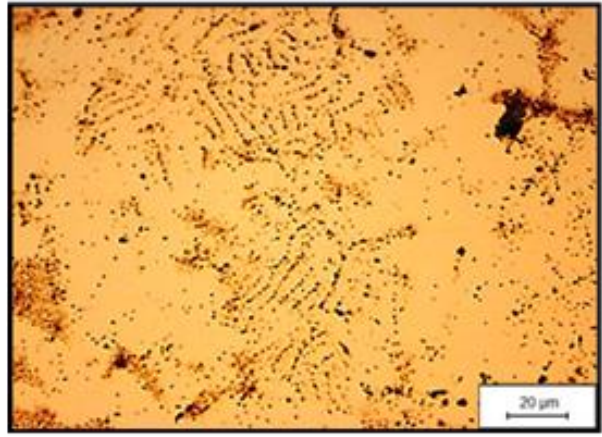

(c)

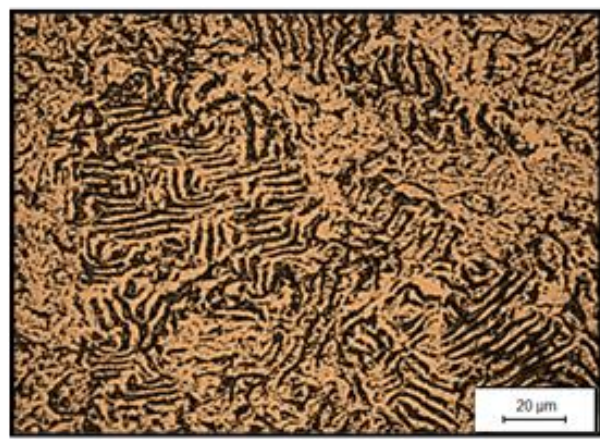

(e)

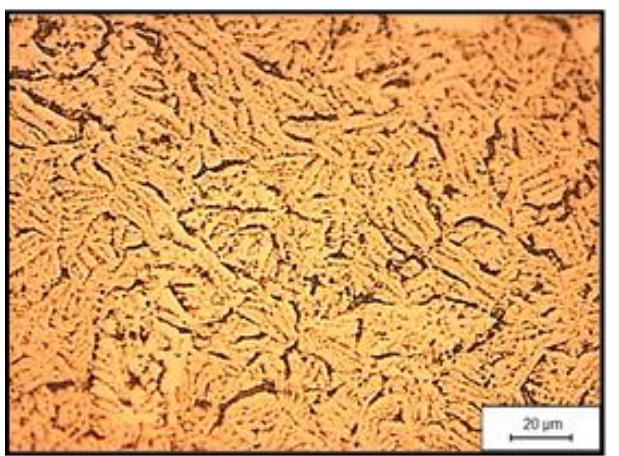

(g)

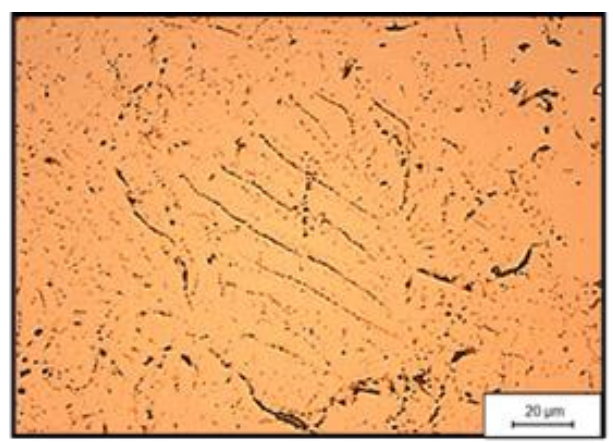

(b)

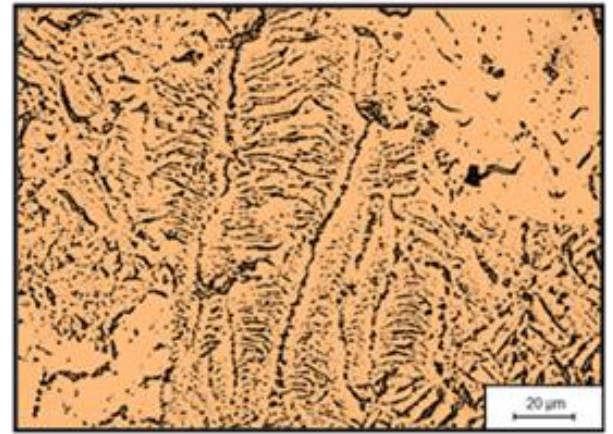

(d)

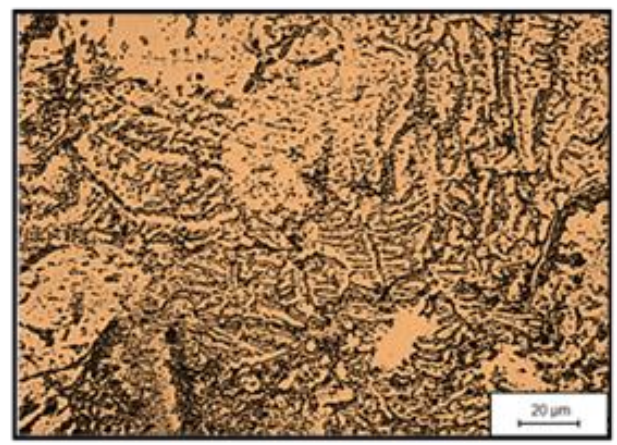

(f)

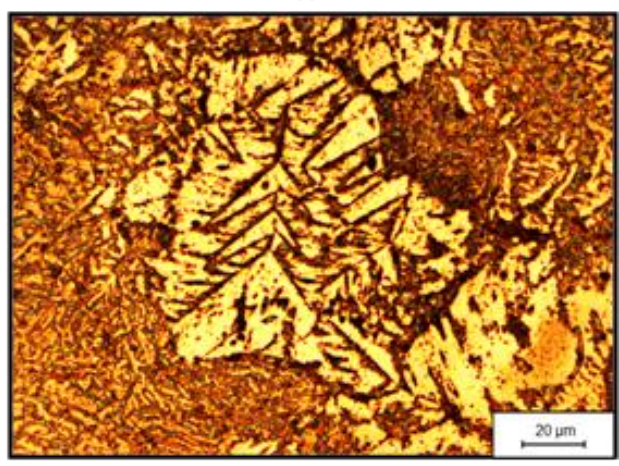

(h)

Figura 6: Figuras (a), (c), (e) e (g) representam a informação magnética da amostra ASTM A366 antes da deformação plástica. Figuras (b), (d), (f) e (h) representam a informação magnética da amostra ASTM A366 após da deformação plástica.

Ao observar as áreas destacadas na Figura 5 (com ferrofluido) em maior magnificação, ou seja, figura 6 (b), e compara-la com imagens feitas da informação magnética da mesma amostra antes da deformação, figura 6 (a), tem-se que, após a 
deformação plástica, os domínios magnéticos apresentam uma variação em seu tamanho (distância entre duas paredes de Bloch). Cerca de 200 imagens antes e após deformação plástica foram analisadas percebendo-se que os domínios magnéticos antes da deformação plástica possuíam um tamanho de $(3 \pm 1) \mu \mathrm{m}$ e após a deformação plástica o tamanho de domínio magnético passou a ser de $(8 \pm 2)$ $\mu \mathrm{m}$.

As figuras 6 (c), (e) e (g) representam as informações magnéticas das áreas a serem deformadas, já as imagens (d), (f) e (h) da figura 6 representam as informações magnéticas destas áreas após a deformação plástica. Ao compará-las percebe-se que, após a deformação plástica, os domínios magnéticos assumem uma estrutura de espinha de peixe, onde temos aglomerações de partículas formando uma linha central e finas paredes de Bloch com suaves inclinações ao redor desta linha. Ao medir a angulação formada por estas espinhas de peixe temos resultados entre 80 e 90 graus. SCHÄFER reportou em seu livro, "The Analysis of Magnetics Microstructures“, um estudo sobre crescimento de domínios magnéticos pela aplicação de campos magnéticos mostrando que, após o crescimento destes domínios, percebe-se também estruturas similares as espinhas com angulações entre 80 e 120 graus, assim como apresentado neste trabalho após a deformação plástica. Em especial na figura 6 (h) vemos a formação de uma conhecida estrutura de árvore de natal [13].

\section{CONCLUSÃO}

Dada a motivação inicial deste trabalho em torno de explicar a modificação da distribuição e intensidade do campo magnético em uma amostra após deformação plástica devido à mudança nos domínios magnéticos, observou-se através dos ensaios com ferrofluido que os domínios magnéticos após deformação plástica se modificam não apenas em seu tamanho, mas também em sua morfologia. Além da identificação da modificação dos domínios magnéticos através da deformação plástica, o ensaio com ferrofluido mostrou-se eficiente para a revelação de informação magnética nos aços estudados.

Percebeu-se que a concentração de carbono presente nas amostras influencia a observação dos domínios magnéticos das amostras, de modo que, quanto maior a concentração de carbono, maior é a dificuldade na observação dos domínios magnéticos da ferrita. Isto ocorre, pois, o carbono é um elemento formador de fases ferromagnéticas, como, por exemplo, a cementita, gerando assim uma maior disputa magneto-atrativa das partículas superparamagnéticas presentes no ferrofluido, o que proporciona um menor contraste nas imagens capturadas;

Observou-se que as informações magnéticas capturadas nas imagens através de ensaio com ferrofluido correlacionam-se com a microestrutura convencional do material. As partículas superparamagnéticas podem ser reveladoras de microconstituintes ferromagnéticos. Um exemplo é o caso mostrado da perlita, onde as partículas superparamagnéticas se aglomeram na região perlítica que possui a cementita, que por sua vez é portadora de um caráter ferromagnético maior que o da matriz ferrítica. Além disso, é possível delimitar as regiões de contorno de grão através de ensaio com ferrofluido, uma vez que os domínios se distribuem no interior dos grãos e podem mudar sua orientação ao passar de um grão para outro, gerando, portanto, uma região de transição denominada parede de Bloch, onde as partículas reagem à informação magnética emergente desta região aglomerando-se e tornando-a visível. 
O ensaio com ferrofluido pode ser utilizado para revelar, de forma macroscópica, o histórico de deformação plástica sofrida pelo material, uma vez que as partículas superparamagnéticas aglomeram-se em maior quantidade nas regiões deformadas plasticamente, de modo a determinar o local onde a amostra fora deformada anteriormente.

\section{Agradecimentos}

Os autores agradecem à Capes e ao CNPq pelo apoio financeiro e a BAM pela oportunidade de trabalho.

\section{REFERÊNCIAS}

[1] Echeverri, E. A. A. (2012) Análise numérica e experimental das tensões residuais geradas durante o processo de têmpera de cilindros de aço AISI 1045, 4140 e 4340. 185f. Dissertação (Mestrado em Engenharia Metalúrgica e de Materiais). Universidade de São Paulo, São Paulo.

[2] J. Pal'a, Magnetic Behaviour of low-carbon steel in parallel and perpendicular directions to tensile Deformation, J. Magn. Magn. Matter, 310, 57-62 (2007).

[3] Dubov, A.A., A study of metal properties using the method of magnetic memory. Metal Science and Heat Treatment, 1997. 39(9-10): p. 401-405.

[4] Nadja Sonntag. Nondestructive Detection of Mechanically Induced Magnetic Stray Fields in Steels. Junior Euromat. 2014.

[5] Kida, K., Effect of plastic Deformation on magnetic fields around fatigue cracks tips of carbono tool steel (JIS, SKS93). International Journal of Fatique, 88, 156-165 (2016)

[6] Sonntag, N. Stegmann, R. Self-magnetic-leakage field detection using magnetooptical sensor technique. MATEC Web of Conferences 12, 04010. 2014

[7] Pelkner, M. Entwicklung, Untersuchung und Anwendung von GMR-Sensorarrays für die Zerstörungsfreie Prüfung von ferromagnetischen Bauteilen. Dissertation zur Erlangung des Grades des Doktors der Ingenieurwissenschaften der Naturwissenschaftlich-Technischen Fakultät II - Physik und Mechatronik - der Universität des Saarlandes 2014

[8] J. Talonen, P. Aspegren and H. Hänninen, Comparison of different methods for measuring strain induced $\alpha^{\prime}$-martensite content in austenitic steels. Materials Science and Technology December 2004 Vol. 20

[9] O. Quintana, "Structural and Magnetic Studies of an Ex-service Cast Austenitic Steel Tube Used in Hydrogen Reformers," University of Illinois at Chicago, 2012.

[10] F. Bitter, "Experiments on the nature of ferromagnetism," Phys. Rev., vol. 41, pp. 507-515, 1932.

[11] Russel, W.B., Saville, D.A., Schowalter, W.R., 1989, Colloidal Dispersions, Cambridge

[12] S.W.J. Smith; W. White; S.G. Barker (1911). "The Magnetic Transition Temperature of Cementite". Proc. Phys. Soc. London 24 (1): 62-69.

[13] Rudolf SCHÄFER e Alex Hubert. "Magnetic Domains The Analysis of Magnetics Microstructures". 5ed. Dresden. Springer. 2009. 